\title{
Respiratory outcomes after six months of hospital discharge in patients affected by COVID-19: a prospective cohort
}

\section{Gabriele da Silveira Prestes}

Universidade do Extremo Sul Catarinense

\section{Carla Sasso Simon}

Universidade do Extremo Sul Catarinense

\section{Roger Walz}

Universidade Federal de Santa Catarina

\section{Cristiane Ritter}

Universidade do Extremo Sul Catarinense

Felipe Dal Pizzol ( $\nabla$ fdpizzol@gmail.com )

Universidade do Extremo Sul Catarinense https://orcid.org/0000-0003-3003-8977

\section{Research}

Keywords: Pulmonary function, COVID-19, Long-COVID, activities of daily living

Posted Date: September 23rd, 2021

DOI: https://doi.org/10.21203/rs.3.rs-915747/v1

License: (c) (i) This work is licensed under a Creative Commons Attribution 4.0 International License. Read Full License

Version of Record: A version of this preprint was published at Frontiers in Medicine on March 7th, 2022. See the published version at https://doi.org/10.3389/fmed.2022.795074. 


\section{Abstract \\ Background}

Considering millions of people affected by COVID-19, long-lasting sequelae can significantly impact health worldwide. Data from prospective studies in lower-middle-income countries on persistent lung dysfunction secondary to COVID-19 are lacking. This work aims to determine risk factors and the impact of persistent lung dysfunctions in COVID-19 survivors.

\section{Methods}

observational and prospective cohort of patients admitted to a tertiary hospital from June 2020 to November 2020. Persistence of chest computed tomography (CT) scan alterations, desaturation in the six-minute walk test (6MWT), forced expiratory volume in one second (FEV1), lung carbon monoxide diffusion (DLCO), and maximum inspiratory pressure (MIP) were measured six months after hospital discharge. Additionally, the Barthel index (BI) and the Modified Medical Research Council (mMRC) Dyspnea Scale were used to determine the impact of lung dysfunction in activities of daily living (ADL).

\section{Results}

It was included 44 patients. Sixty percent had persistent lung CT scan abnormalities. From 18 to 43 percent of patients had at least one pulmonary function dysfunction, a decrease in FEV1 was the least prevalent (18\%), and a reduction in DCLO and MIP was the most frequent (43\%). In general, female gender, comorbidity index, and age were associated with worse lung function. Additionally, the presence of lung dysfunction could predict worse BI (r-square 0.28) and mMRC (r-square 0.32).

\section{Conclusion}

Long-term lung dysfunction is relatively common in survivors from severe COVID-19 and impacts negatively on ADL and the intensity of dyspnea, similar to studies in high-income countries.

\section{Background}

In addition to the characteristic symptoms of the acute infectious process of COVID-19, including fever, cough, and chest discomfort and, in severe cases, dyspnea and bilateral pulmonary infiltration $(1,2)$, "long-COVID" reports are increasing. Still, its prevalence, risk factors, or whether one can predict the occurrence of "long-COVID" is not known $(3,4)$. The consequences of acute lung damage drove by COVID19 could be permanent lung damage if the patient recovers $(5,6)$. 
Until today there is not sufficient evidence on the long-term prognosis of patients who had pneumonia due to COVID-19 (5). In this context, McGroder et al. (2021) (6) evaluated patients four months after hospitalization. Predominantly in patients who underwent mechanical ventilation, fibrotic-type patterns were observed on computed tomography (CT). Elderly patients are at an even higher risk, and in this population, even less severe dysfunctions can cause increased morbidity and mortality. (7) Few studies suggested that this pattern persist up to 12 months after hospitalization (8-10)

Thus, lung dysfunction is a critical question in "long-COVID". Still, we do not have enough data on risk factors, the impact of this dysfunction on patients' daily living, mainly coming from lower-middle-income countries. In this context, we performed a prospective cohort study to identify pulmonary outcomes after six months of hospital discharge in patients who developed pneumonia due to COVID-19 in South Brazil. We hypothesize that six months after hospitalization due to COVID-19 pneumonia, lung dysfunction is frequent and negatively affects the activities of daily living (ADL).

\section{Methods}

\section{Study design}

A prospective cohort was conducted with patients admitted to a tertiary hospital from June 2020 to November 2020. The São José hospital's Institutional Review Board approved the protocol under the number 31384620.6.1001.5364. All patients or their surrogates gave written consent before inclusion in the study.

\section{Setting}

The study sample consisted of all consecutive patients admitted to the COVID-19 ward or intensive care unit (ICU) of a tertiary hospital from June 2020 to November 2020. The inclusion criteria were patients over 18 years old admitted to the hospital with confirmed COVID-19 diagnosis through reverse transcriptase reaction or rapid antigen test and requiring supplementary oxygen, non-invasive ventilation, or mechanical ventilation due to COVID-19 pneumonia. Exclusion criteria were patients with severe chronic diseases or diseases capable of altering inflammatory response, such as chronic use of immunosuppressants, cancer patients without disease control, chronic kidney disease under dialysis, Cirrhosis Child C, HIV, severe COPD, severe heart failure, and patients in palliative care or with life expectancy $<24$ hours.

\section{Procedures}

Investigators daily screened all patients admitted to the hospital, and those who met the inclusion criteria were considered eligible. The patient was invited to participate in the study from hospital admission for a maximum of 120 hours. All necessary information was prospectively collected directly from the patient's electronic medical record. Prehospital comorbidities were aggregated through the validated Charlson comorbidity index. The severity of critical illness at ICU admission was collected with the Simplified Acute Physiology Score (SAPS) 3. The Sequential Organ Failure Assessment (SOFA) score was used to assess 
organ dysfunction. A chest CT scan was performed on admission and was analyzed to determine the extent of pulmonary involvement. Six months after hospital discharge, patients attended the hospital's outpatient clinic. They were evaluated with chest CT scan, lung carbon monoxide diffusion (DLCO) and lung plethysmography, a 6-minute walk test (6MWT), $A D L$, and dyspnea intensity, performed as described below. The outcome evaluation was blinded for the hospitalization variables.

\section{Chest Computed Tomography}

The extension of acute-phase ground-glass opacity was graded as less than $25 \%, 25$ to $50 \%$, and greater than $50 \%$ at hospital admission. At 6 -months after hospital discharge, the persistence of ground-glass and the occurrence of lung fibrosis were evaluated.

\section{Lung plethysmography with carbon monoxide diffusion}

A variable pressure VIASYS Respiratory Care plethysmography measured lung volumes and DLCO. This study used forced expiratory volume in one second (FEV1), DLCO, and maximum inspiratory pressure (MIP) to assess lung function. These variables were expressed as percentages of predicted normal values. Normal values were considered those equal to or greater than $80 \%$ of predicted values.

\section{Six-minute walk test (6MWT)}

Each patient walked on the flat ground as fast as possible without oxygen inhalation and completed the $6 \mathrm{MWT}$ independently. From the 6MWT, significant oxygen desaturation was the parameter used to qualify the patient's performance, defined as a decrease of at least $4 \%$ from baseline $\mathrm{SpO}_{2}$.

\section{The Barthel Index}

The Barthel Index $(\mathrm{BI})$ is a ten-item ordinal scale used to measure performance ADL. BI scored according to the level of physical assistance required to perform the daily task.

\section{The Modified Medical Research Council Dyspnea Scale}

The Modified Medical Research Council (mMRC) Dyspnea Scale is a self-rating tool to measure the degree of disability that breathlessness poses on day-to-day activities on a scale from 0 to 4 .

\section{Outcomes}

Outcomes were different aspects of lung function 6-months after hospital discharge: persistence of CT scan alterations, desaturation in the 6MWT, FEV1, DLCO, and MIP. Additionally, the BI and the mMRC were used to determine the impact of lung dysfunction in ADL.

\section{Statistical Analysis}

The collected data were analyzed in the IBM Statistical Package for Social Sciences (SPSS) version 22.0 software. Quantitative variables were expressed as mean and standard deviation, and were compared using the Student's t-test. Nominal variables were expressed as frequency and percentage, and were compered using the Pearson's chi-square. The binary regression was used to access the independent risk 
factors for outcomes. The model included only variables with $p<0.20$ or $p<0.05$ in the univariate analysis depending on the number of events observed in each outcome to not overfitting the model. If variables that reached the threshold had collinearity, the variable with a lower $p$-value in the univariate analysis entered the final model. Results from univariate analysis were presented as p-value and binary regression as relative risk and $95 \%$ confidence interval. Linear regression was performed to determine the impact of lung dysfunction on $A D L$, and R squared was calculated to express the percentage of the variance in the $A D L$ that the lung dysfunction variables explained. In all analyses, a p-value $<0.05$ was adopted as the level for statistical significance.

\section{Results}

Based on the predefined inclusion and exclusion criteria, the final sample resulted in 167 patients. From these, 56 patients died during hospitalization or follow-up. Due to the importance of a timely description of lung abnormalities, we evaluated only the first 44 consecutively included patients six months after hospital discharge. There were no missing cases. All patients will have a 1-year evaluation, as defined in the original protocol.

Table 1 described demographic information. $70 \%$ of the patients were male, and the mean age was 54 years. The mean body mass index was 30 , and the mean Charlson comorbidity index was 1.63 . The mean length of ICU stay was 12 days and the mean length of hospital stay was 16 days. The need for mechanical ventilation was $23 \%$ of the sample. The mean SAPS III score was 48 , and the mean respiratory SOFA was 2.8 at admission (D1) and 2.072 hours after (D3). These variables were not statistically different compared to the remaining 67 patients not included in this preliminary analysis. 
Table 1

- General patients' characteristics

\begin{tabular}{|c|c|c|}
\hline Variables & $N(\%)$ & Mean (SD) \\
\hline Age, & & $54(11)$ \\
\hline \multicolumn{3}{|l|}{ Years } \\
\hline Gender, & $31(70)$ & \\
\hline Male & 13(30) & \\
\hline \multicolumn{3}{|l|}{ Female } \\
\hline BMI & & $30(5.0)$ \\
\hline Extent of lung involvement at admission & $15(40)$ & \\
\hline 25 to $50 \%$ & $23(60)$ & \\
\hline \multicolumn{3}{|l|}{$>50 \%$} \\
\hline Charlson Comorbidity Index & & $1.63(1.2)$ \\
\hline Corticosteroids, yes & $40(91)$ & \\
\hline SAPS III & & $48(12.2)$ \\
\hline Respiratory SOFA & & $2.8(1.0)$ \\
\hline D1 & & $2.0(1.4)$ \\
\hline \multicolumn{3}{|l|}{ D3 } \\
\hline SOFA & & $3.7(1.9)$ \\
\hline D1 & & $2.9(2.6)$ \\
\hline \multicolumn{3}{|l|}{ D3 } \\
\hline C-reactive protein, $\mathrm{mg} / \mathrm{L}$ & & 118(84) \\
\hline D1 & & 108(96) \\
\hline \multicolumn{3}{|l|}{ D3 } \\
\hline ICU admission & $31(70)$ & \\
\hline Yes & $13(30)$ & \\
\hline \multicolumn{3}{|l|}{ No } \\
\hline ICU length of stay, days & & 12(9.1) \\
\hline
\end{tabular}

SD - standard deviation; BMI - Body mass index; SAPS III - Simplified Acute Physiology Score 3; SOFA sequential organ failure assessment score; ICU - intensive care unit; $\mathrm{N}$ - number of participants. 


\begin{tabular}{|llc|}
\hline Variables & N(\%) & Mean (SD) \\
\hline Invasive mechanical ventilation & $10(23)$ & \\
Yes & $34(77)$ & \\
No & & $13(7.7)$ \\
\hline Days on Mechanical Ventilation & $16(10.3)$ \\
\hline Length of hospital stay, & \\
Days. & & \\
\hline $\begin{array}{l}\text { SD - standard deviation; BMI - Body mass index; SAPS III - Simplified Acute Physiology Score 3; SOFA - } \\
\text { sequential organ failure assessment score; ICU - intensive care unit; N - number of participants. }\end{array}$ \\
\hline
\end{tabular}

Lung CT scan abnormalities were ground-glass (15 from 24) and fibrosis (9 from 24). Thus 24 (60\%) of the patients had persistent lesions on CT-scan. Table 2 presented the relation of acute-phase variables and the persistence of lung abnormalities on the CT-scan 6-months after hospital discharge. In the univariate analysis, no single variable was associated with CT-scan lesions. However, c-reactive protein (CRP) at D1 and D3 and respiratory SOFA at D3 reached the threshold and were included in the regression analysis. Only CRP levels were marginally, but not significantly, related to the persistence of CT-scan lesions (Table 2). 
Table 2

Variables associated with the persistence of lesions on lung computed tomography scan 6-months after hospital discharge.

\begin{tabular}{|c|c|c|c|c|}
\hline Variables & $\begin{array}{l}\text { Present }(n= \\
24)\end{array}$ & $\begin{array}{l}\text { Absence }(n= \\
20)\end{array}$ & $\begin{array}{l}\text { p- } \\
\text { value }\end{array}$ & $\operatorname{RR}(\mathrm{Cl} 95 \%)^{\mathrm{b}}$ \\
\hline Age, years, mean (SD) & $54(11)$ & $54(11)$ & 0.99 & NA \\
\hline Gender & $16(66)$ & $15(75)$ & 0.55 & NA \\
\hline \multicolumn{5}{|l|}{ Male. n (\%) } \\
\hline \multirow{2}{*}{\multicolumn{5}{|c|}{$\begin{array}{l}\text { BMI } \\
\text { mean (SD) }\end{array}$}} \\
\hline & & & & \\
\hline \multirow{3}{*}{$\begin{array}{l}\text { Extent of lung involvement at } \\
\text { admission }{ }^{c} \\
n(\%)\end{array}$} & $8(38)$ & $7(41)$ & 0.85 & NA \\
\hline & $13(62)$ & 10(59) & & \\
\hline & & & & \\
\hline \multicolumn{5}{|l|}{25 to $50 \%$} \\
\hline \multicolumn{5}{|l|}{$>50 \%$} \\
\hline Charlson Comorbidity Index & $1.8(1.2)$ & $1.4(1.2)$ & 0.34 & NA \\
\hline \multicolumn{5}{|l|}{ mean (SD) } \\
\hline SAPS III & $47(14.4)$ & $48(10.6)$ & 0.91 & NA \\
\hline \multicolumn{5}{|l|}{ mean (SD) } \\
\hline Respiratory SOFA & $2.9(1.1)$ & $2.7(0.9)$ & 0.56 & NA \\
\hline D1. mean (SD) & $2.4(1.3)$ & $1.8(1.5)$ & $0.16^{*}$ & $1.04(0.7-$ \\
\hline \multicolumn{5}{|l|}{ D3. mean (SD) } \\
\hline SOFA & $3.6(1.7)$ & $3.9(2.2)$ & 0.59 & NA \\
\hline D1. Mean (SD) & $3.0(2.5)$ & $2.7(2.8)$ & 0.67 & NA \\
\hline \multicolumn{5}{|l|}{ D3. Mean (SD) } \\
\hline C-reactive protein, $\mathrm{mg} / \mathrm{L}$ & $99(72)$ & $146(96)$ & 0.13 & NA \\
\hline D1. Mean (SD) & $79(54)$ & 164(133) & $0.08 *$ & $0.98(0.98-$ \\
\hline D3. Mean (SD) & & & & \\
\hline
\end{tabular}

SD - standard deviation; RR- Relative Risk; $\mathrm{Cl}$ - confidence interval; NA - not applied; BMI - Body mass index; SAPS III - Simplified Acute Physiology Score 3; SOFA - sequential organ failure assessment score; ICU - intensive care unit; $\mathrm{N}$ - number of participants; ${ }^{*} \mathrm{p}<0.20$ variables included in the binary logistic regression. ${ }^{a} \mathrm{p}$-value from the univariate analysis. ${ }^{\mathrm{b}} \mathrm{RR}$ form the regression analysis. ${ }^{\mathrm{C}}$ for six patients, CT scan, was not performed at hospital admission. 


\begin{tabular}{|c|c|c|c|c|}
\hline Variables & $\begin{array}{l}\text { Present }(n= \\
24)\end{array}$ & $\begin{array}{l}\text { Absence }(n= \\
20)\end{array}$ & $\begin{array}{l}\text { p- } \\
\text { value }^{a}\end{array}$ & $\mathrm{RR}(\mathrm{Cl} 95 \%)^{\mathrm{b}}$ \\
\hline \multicolumn{4}{|l|}{ yes, n(\%) } & NA \\
\hline $\begin{array}{l}\text { ICU length of stay, days } \\
\text { mean (SD) }\end{array}$ & 12(9.9) & $11(8.3)$ & 0.65 & NA \\
\hline Mechanical ventilation yes, n(\%) & $4(40)$ & $6(60)$ & 0.29 & NA \\
\hline $\begin{array}{l}\text { Days on Mechanical Ventilation } \\
\text { mean (SD) }\end{array}$ & $14(8.2)$ & 12(7.9) & 0.63 & NA \\
\hline $\begin{array}{l}\text { Length of hospital stay Days. Mean } \\
\text { (SD) }\end{array}$ & $17(11.4)$ & $15(8.9)$ & 0.61 & NA \\
\hline \multicolumn{5}{|c|}{ 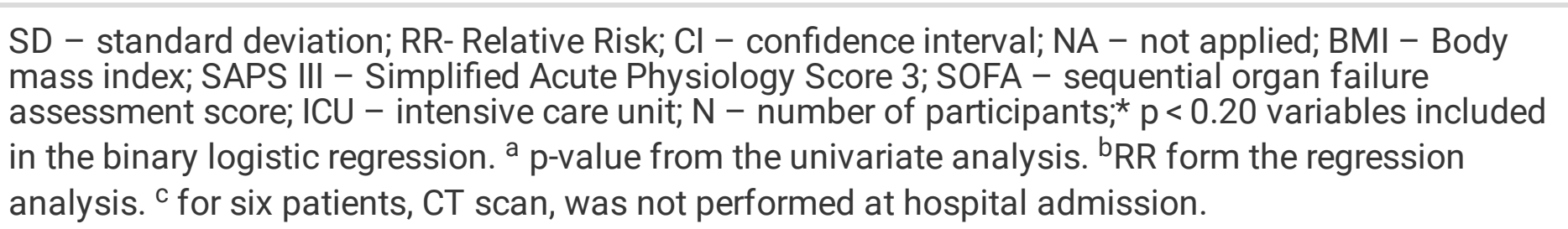 } \\
\hline
\end{tabular}

In the 6MWT (Table 3), 15 (35\%) patients had a significant desaturation. In the univariate analysis, desaturation was associated with female gender, Charlson comorbidity index, and respiratory SOFA D3. Only the female gender was independently associated with desaturation in 6MWT. Of these 15 patients, $10(66 \%)$ had persistence of CT scan alterations, being $6(60 \%)$ ground-glass and 4 (40\%) fibrotic lesions. 
Table 3

Variables associated with desaturation in the 6-minute walk test 6-months after hospital discharge.

\begin{tabular}{|c|c|c|c|c|}
\hline Variables & $\begin{array}{l}\text { Present }(n= \\
15)\end{array}$ & $\begin{array}{l}\text { Absence }(n= \\
29)\end{array}$ & $\begin{array}{l}\mathrm{p}- \\
\text { value }^{\mathrm{a}}\end{array}$ & RR (IC 95\%) \\
\hline \multicolumn{5}{|l|}{ years, mean(SD) } \\
\hline \multicolumn{5}{|l|}{ Male. n (\%) } \\
\hline \multicolumn{4}{|l|}{ mean (SD) } & NA \\
\hline $\begin{array}{l}\text { Extent of lung involvement at } \\
\text { admissionc } \\
n(\%) \\
25 \text { to } 50 \% \\
>50 \%\end{array}$ & $\begin{array}{l}4(27) \\
11(73)\end{array}$ & $\begin{array}{l}12(50) \\
12(50)\end{array}$ & 0.08 & NA \\
\hline $\begin{array}{l}\text { Charlson Comorbidity Index } \\
\text { mean (SD) }\end{array}$ & $2.1(1.2)$ & $1.4(1.1)$ & $0.044^{\star}$ & $1.5(0.8-2.9)$ \\
\hline $\begin{array}{l}\text { SAPS III } \\
\text { mean (SD) }\end{array}$ & $53(15.0)$ & $46(10.1)$ & 0.15 & NA \\
\hline Respiratory SOFA & $2.9(1.2)$ & $2.7(0.9)$ & 0.50 & NA \\
\hline $\begin{array}{l}\text { D1. mean (SD) } \\
\text { D3. mean (SD) }\end{array}$ & $2.6(1.1)$ & $1.8(1.5)$ & $0.045^{\star}$ & $1.06(0.8-1.4)$ \\
\hline SOFA & $3.6(1.7)$ & $3.6(1.7)$ & 0.73 & NA \\
\hline $\begin{array}{l}\text { D1. mean (SD) } \\
\text { D3. mean (SD) }\end{array}$ & $3.6(2.8)$ & $2.4(2.4)$ & 0.15 & NA \\
\hline C-reactive protein, $\mathrm{mg} / \mathrm{L}$ & $88(45)$ & $136(96)$ & 0.07 & NA \\
\hline $\begin{array}{l}\text { D1. mean (SD) } \\
\text { D3. mean (SD) }\end{array}$ & $91(89)$ & $122(102)$ & 0.40 & NA \\
\hline $\begin{array}{l}\text { SD - standard deviation; RR- Re } \\
\text { index; SAPS III - Simplified Acu } \\
\text { score; ICU - intensive care unit; } \\
\text { regression. a p-value from the } \\
\text { CT scan was not performed at }\end{array}$ & $\begin{array}{l}\text { k; Cl - confider } \\
\text { ogy Score } 3 ; \mathrm{S} \\
\text { er of participai } \\
\text { analysis. }{ }^{\text {b RR }} \\
\text { dmission. }\end{array}$ & $\begin{array}{l}\text { interval; } N A-n c \\
A-\text { sequential } c \\
k<<0.20 \text { and } i \\
n \text { the regressic }\end{array}$ & $\begin{array}{l}\text { pplied; B } \\
\text { n failure } \\
\text { uded in th } \\
\text { nalysis. }\end{array}$ & $\begin{array}{l}\text { - Body mass } \\
\text { ssessment } \\
\text { binary logistic } \\
\text { or five patients, }\end{array}$ \\
\hline
\end{tabular}




\begin{tabular}{|c|c|c|c|c|}
\hline Variables & $\begin{array}{l}\text { Present }(n= \\
15)\end{array}$ & $\begin{array}{l}\text { Absence }(n= \\
29)\end{array}$ & $\begin{array}{l}\text { p- } \\
\text { value }\end{array}$ & RR (IC 95\%) ${ }^{b}$ \\
\hline $\begin{array}{l}\text { ICU } \\
\text { yes, } n(\%)\end{array}$ & $11(73)$ & $20(69)$ & 0.76 & NA \\
\hline \multicolumn{5}{|l|}{ mean $(S D)$} \\
\hline $\begin{array}{l}\text { Mechanical Ventilation } \\
\text { yes, } n(\%)\end{array}$ & $4(27)$ & $6(21)$ & 0.65 & NA \\
\hline $\begin{array}{l}\text { Days on Mechanical Ventilation } \\
\text { mean (SD) }\end{array}$ & $15(5.3)$ & $11(9.2)$ & 0.750 & NA \\
\hline \multicolumn{4}{|l|}{ Days. mean (SD) } & NA \\
\hline $\begin{array}{l}\text { SD - standard deviation; RR- Rela } \\
\text { index; SAPS III - Simplified Acute } \\
\text { score; ICU - intensive care unit; } N \\
\text { regression. }{ }^{\text {a }} \text { p-value from the un } \\
\text { CT scan was not performed at h }\end{array}$ & $\begin{array}{l}\mathrm{k} ; \mathrm{Cl} \text { - confider } \\
\text { logy Score 3; } \mathrm{S} \\
\text { er of participa } \\
\text { analysis. }{ }^{\mathrm{b}} \mathrm{RR} \\
\text { admission. }\end{array}$ & $\begin{array}{l}\text { interval; NA - no } \\
A-\text { sequential o } \\
{ }^{\star} p<0.20 \text { and ir } \\
m \text { the regressio }\end{array}$ & $\begin{array}{l}\text { pplied; } \mathrm{B} \\
\text { n failure } \\
\text { uded in th } \\
\text { nalysis. }\end{array}$ & $\begin{array}{l}\text { - - Body mass } \\
\text { ssessment } \\
\text { binary logistic } \\
\text { or five patients, }\end{array}$ \\
\hline
\end{tabular}

Only $8(18 \%)$ of the patients had a significant ( $<80 \%$ of predicted value) decrease in FEV1. When analyzing the FEV1 (Table 4), age was significantly associated with an abnormal FEV1. Interestingly, all patients that had reduced FEV1 had more than $50 \%$ of ground-glass at hospital admission. However, due to the low number of events, every attempt to perform a binary regression resulted in an overfitted model. Of these eight patients, $2(25 \%)$ had persistence of CT scan alterations, being 1 (50\%) ground-glass and 1 $(50 \%)$ fibrotic lesions. 
Table 4

Variables associated with a decreased forced expiratory volume in one second 6-months after hospital discharge.

\begin{tabular}{|c|c|c|c|}
\hline Variables & Present $(n=8)$ & Absence $(n=36)$ & p-value ${ }^{a}$ \\
\hline $\begin{array}{l}\text { Age } \\
\text { years. mean(SD) }\end{array}$ & $60(5)$ & $53(11)$ & $0.015^{\star}$ \\
\hline $\begin{array}{l}\text { Gender } \\
\text { Male. n (\%) }\end{array}$ & $6(75)$ & $25(10)$ & 0.75 \\
\hline $\begin{array}{l}\text { BMI } \\
\text { mean (SD) }\end{array}$ & $32(5.4)$ & $30(5.0)$ & 0.35 \\
\hline $\begin{array}{l}\text { Extent of lung involvement at admission }{ }^{\text {b }} \\
n(\%) \\
25 \text { to } 50 \% \\
>50 \%\end{array}$ & $\begin{array}{l}0(0.0) \\
7(100)\end{array}$ & $\begin{array}{l}15(48) \\
16(52)\end{array}$ & $0.029 *$ \\
\hline $\begin{array}{l}\text { Charlson Comorbidity Index } \\
\text { mean (SD) }\end{array}$ & $2.1(0.8)$ & $1.5(1.2)$ & 0.11 \\
\hline $\begin{array}{l}\text { SAPS III } \\
\text { mean (SD) }\end{array}$ & $50(15.2)$ & $48(12.0)$ & 0.71 \\
\hline Respiratory SOFA & $2.9(1.2)$ & $2.8(0.9)$ & 0.80 \\
\hline $\begin{array}{l}\text { D1. mean (SD) } \\
\text { D3. mean (SD) }\end{array}$ & $2.0(1.4)$ & $2.1(1.4)$ & 0.87 \\
\hline SOFA & $3.9(2.8)$ & $3.7(1.7)$ & 0.81 \\
\hline $\begin{array}{l}\text { D1. mean (SD) } \\
\text { D3. mean (SD) }\end{array}$ & $2.6(2.1)$ & $2.9(2.8)$ & 0.76 \\
\hline C-reactive protein, $\mathrm{mg} / \mathrm{L}$ & 108(103) & $122(80)$ & 0.70 \\
\hline D1. mean (SD) & 129(172) & 103(70) & 0.72 \\
\hline D3. mean (SD) & & & \\
\hline
\end{tabular}

SD - standard deviation; BMI - Body mass index; FEV1 - forced expiratory volume in one second; SAPS III - Simplified Acute Physiology Score 3; SOFA - sequential organ failure assessment score; ICU intensive care unit; $\mathrm{N}$ - number of participants. ${ }^{a} \mathrm{p}$-value from the univariate analysis. ${ }^{\mathrm{b}}$ for six patients CT scan was not performed at hospital admission. * Due to the low number of events, it was not possible to perform binary regression analysis. 


\begin{tabular}{|c|c|c|c|}
\hline Variables & Present $(n=8)$ & Absence $(n=36)$ & p-value ${ }^{a}$ \\
\hline $\begin{array}{l}\text { ICU } \\
\text { yes, } n(\%)\end{array}$ & $5(62)$ & $26(72)$ & 0.59 \\
\hline \multicolumn{3}{|l|}{ mean $(S D)$} & 0.51 \\
\hline $\begin{array}{l}\text { Mechanical Ventilation } \\
\text { yes, } n(\%)\end{array}$ & $2(25)$ & $8(22)$ & 0.86 \\
\hline $\begin{array}{l}\text { Days on Mechanical Ventilation } \\
\text { mean (SD) }\end{array}$ & 18(7.8) & $11(7.4)$ & 0.25 \\
\hline $\begin{array}{l}\text { Length of hospital stay } \\
\text { Days. mean (SD) }\end{array}$ & 19(11.3) & $15(10.0)$ & 0.35 \\
\hline $\begin{array}{l}\text { SD - standard deviation; BMI - Boc } \\
\text { III - Simplified Acute Physiology S } \\
\text { intensive care unit; } \mathrm{N} \text { - number of } \\
\text { CT scan was not performed at ho } \\
\text { possible to perform binary regress }\end{array}$ & $\begin{array}{l}\text { EV1 - forced exp } \\
\text { equential organ } f \\
\text {-value from the } u \\
\text { h. * Due to the lov }\end{array}$ & $\begin{array}{l}\text { tory volume in one } \\
\text { ure assessment scc } \\
\text { lariate analysis. }{ }^{b} \mathrm{f} \\
\text { umber of events, it }\end{array}$ & $\begin{array}{l}\text { ond; SAPS } \\
\text { ICU- } \\
\text { ix patients } \\
\text { s not }\end{array}$ \\
\hline
\end{tabular}

When analyzing DLCO (Table 5), 19 (43\%) of patients presented a significant decrease. In the univariate analysis age, gender and Charlson comorbidity index were significantly associated with a reduction in DLCO, but only gender was independently associated with a decrease in diffusion. Of these 19 patients, 11 (58\%) had persistence of CT scan alterations, being 6 (54\%) ground-glass and 5 (36\%) fibrotic lesions. 
Table 5

Variables associated with a decreased carbon monoxide diffusion 6-months after hospital discharge.

\begin{tabular}{|c|c|c|c|c|}
\hline Variables & $\begin{array}{l}\text { Present }(n= \\
\text { 19) }\end{array}$ & $\begin{array}{l}\text { Absence }(n= \\
25)\end{array}$ & $\begin{array}{l}\text { p- } \\
\text { value }^{a}\end{array}$ & $\operatorname{RR}(\text { IC 95\%) })^{b}$ \\
\hline $\begin{array}{l}\text { Age } \\
\text { years. mean(SD) }\end{array}$ & $59(9)$ & $50(11)$ & $0.008^{*}$ & $1.03(0.9-1.2)$ \\
\hline $\begin{array}{l}\text { Gender } \\
\text { Male. n (\%) }\end{array}$ & $9(47)$ & 22(88) & $0.003^{*}$ & $0.7(0.006-0.7)$ \\
\hline $\begin{array}{l}\text { BMI } \\
\text { mean (SD) }\end{array}$ & $30(5.6)$ & $31(4.7)$ & 0.73 & NA \\
\hline $\begin{array}{l}\text { Extent of lung involvement at } \\
\text { admissionc } \\
n(\%) \\
25 \text { to } 50 \% \\
>50 \%\end{array}$ & $\begin{array}{l}5(36) \\
9(64)\end{array}$ & $\begin{array}{l}10(42) \\
14(58)\end{array}$ & 0.72 & NA \\
\hline $\begin{array}{l}\text { Charlson Comorbidity Index, mean } \\
\text { (SD) }\end{array}$ & $2.1(1.3)$ & $1.3(1.0)$ & $0.02^{\star}$ & $1.9(0.65-5.6)$ \\
\hline $\begin{array}{l}\text { SAPS III } \\
\text { mean (SD) }\end{array}$ & $48(10.6)$ & $48(14)$ & 0.88 & NA \\
\hline Respiratory SOFA & $2.68(1.00)$ & $2.88(0.97)$ & 0.51 & NA \\
\hline $\begin{array}{l}\text { D1. mean (SD) } \\
\text { D3. mean (SD) }\end{array}$ & $2.32(1.25)$ & $1.87(1.51)$ & 0.30 & NA \\
\hline SOFA & $3.3(2.2)$ & $4.0(1.6)$ & 0.21 & NA \\
\hline $\begin{array}{l}\text { D1. mean (SD) } \\
\text { D3. mean (SD) }\end{array}$ & $3.0(2.0)$ & $2.8(3.1)$ & 0.88 & NA \\
\hline C-reactive protein, $\mathrm{mg} / \mathrm{L}$ & $96(63)$ & 137(96) & 0.16 & NA \\
\hline $\begin{array}{l}\text { D1. mean (SD) } \\
\text { D3. mean (SD) }\end{array}$ & $83(51)$ & $132(121)$ & $0.16^{*}$ & $\begin{array}{l}0.99(0.97- \\
1.005)\end{array}$ \\
\hline
\end{tabular}

SD - standard deviation; RR- Relative Risk; Cl - confidence interval; NA - not applied; BMI - Body mass index; SAPS III - Simplified Acute Physiology Score 3; SOFA - sequential organ failure assessment score; ICU - intensive care unit; $\mathrm{N}$ - number of participants; ${ }^{a} \mathrm{p}$-value from the univariate analysis. ${ }^{\mathrm{b}} \mathrm{RR}$ form the regression analysis. ${ }^{C}$ for six patients, CT scan was not performed at hospital admission. 


\begin{tabular}{|c|c|c|c|c|}
\hline Variables & $\begin{array}{l}\text { Present }(n= \\
\text { 19) }\end{array}$ & $\begin{array}{l}\text { Absence }(n= \\
25)\end{array}$ & $\begin{array}{l}\mathrm{p}- \\
\text { value }^{\mathrm{a}}\end{array}$ & RR (IC 95\%) ${ }^{b}$ \\
\hline \multicolumn{5}{|l|}{ yes, n(\%) } \\
\hline \multicolumn{5}{|l|}{ mean (SD) } \\
\hline $\begin{array}{l}\text { Mechanical Ventilation } \\
\text { yes, } n(\%)\end{array}$ & $3(16)$ & $7(28)$ & 0.34 & NA \\
\hline $\begin{array}{l}\text { Days on Mechanical Ventilation } \\
\text { mean (SD) }\end{array}$ & $13(5.5)$ & $12(8.8)$ & 0.87 & NA \\
\hline \multicolumn{4}{|l|}{ Days. mean (SD) } & NA \\
\hline $\begin{array}{l}\text { SD - standard deviation; RR- Relat } \\
\text { index; SAPS III - Simplified Acute } \\
\text { score; ICU - intensive care unit; } N \text { - } \\
\text { form the regression analysis. }{ }^{C} \text { fol }\end{array}$ & $\begin{array}{l}\text { sk; Cl - confide } \\
\text { logy Score 3; } \\
\text { er of particip } \\
\text { atients, CT sc }\end{array}$ & $\begin{array}{l}\text { interval; NA - } \\
\text { FA - sequential } \\
s ;{ }^{\text {a }} \text { p-value fron } \\
\text { was not perforr }\end{array}$ & $\begin{array}{l}\text { applied; } \\
\text { yan failu } \\
\text { le univar } \\
\text { at hosp }\end{array}$ & $\begin{array}{l}\text { MI - Body mass } \\
\text { assessment } \\
\text { te analysis. }{ }^{b} \mathrm{RR} \\
\text { l admission. }\end{array}$ \\
\hline
\end{tabular}

MIP assessed respiratory muscle strength and was decreased in 19 (43\%) patients (Table 6). Age, gender, and Charlson comorbidity index were associated with reduced MIP in the univariate analysis, but no one variable was independently associated with this outcome. It was observed a marginal, non-significant association with gender and Charlson comorbidity index. Of these 19 patients, 11 (58\%) had persistence of CT scan alterations, being 7 (64\%) ground-glass and 4 (36\%) fibrotic lesions. 
Table 6

Variables associated with a decreased Maximum Inspiratory Pressure 6-months after hospital discharge.

\begin{tabular}{|c|c|c|c|c|}
\hline Variables & $\begin{array}{l}\text { Present }(n= \\
19)\end{array}$ & $\begin{array}{l}\text { Absence }(\mathrm{n}= \\
25)\end{array}$ & $\begin{array}{l}\text { p- } \\
\text { value }^{a}\end{array}$ & RR (IC 95\%) \\
\hline $\begin{array}{l}\text { Age } \\
\text { years. mean(SD) }\end{array}$ & $57(9.0)$ & $51(11.6)$ & $0.05^{\star}$ & $\begin{array}{l}0.96(0.86- \\
1.08)\end{array}$ \\
\hline $\begin{array}{l}\text { Gender } \\
\text { Male. n (\%) }\end{array}$ & $10(53)$ & $20(80)$ & $0.03^{*}$ & $\begin{array}{l}0.25(0.05- \\
1.16)\end{array}$ \\
\hline $\begin{array}{l}\text { BMI } \\
\text { mean (SD) }\end{array}$ & $30(6.0)$ & $31(4.4)$ & 0.71 & NA \\
\hline $\begin{array}{l}\text { Extent of lung involvement at } \\
\text { admissionc } \\
n(\%) \\
25 \text { to } 50 \% \\
>50 \%\end{array}$ & $\begin{array}{l}7(40) \\
9(60)\end{array}$ & $\begin{array}{l}9(41) \\
13(59)\end{array}$ & 0.96 & NA \\
\hline $\begin{array}{l}\text { Charlson Comorbidity Index } \\
\text { mean (SD) }\end{array}$ & $2.2(1.3)$ & $1.2(1.0)$ & $0.008^{*}$ & $2.6(0.8-8.0)$ \\
\hline $\begin{array}{l}\text { SAPS III } \\
\text { mean (SD) }\end{array}$ & $45(10.0)$ & $50(13.6)$ & 0.36 & NA \\
\hline Respiratory SOFA & $2.8(0.8)$ & $2.7(1.1)$ & 0.65 & NA \\
\hline $\begin{array}{l}\text { D1. mean (SD) } \\
\text { D3. mean (SD) }\end{array}$ & $2.3(1.2)$ & $1.9(1.6)$ & 0.36 & NA \\
\hline SOFA & $3.9(1.6)$ & $3.6(2.2)$ & 0.71 & NA \\
\hline $\begin{array}{l}\text { D1. mean (SD) } \\
\text { D3. mean (SD) }\end{array}$ & $3.0(2.1)$ & $2.9(3.1)$ & 0.78 & NA \\
\hline C-reactive protein, mg/L & 138(70) & $100(97)$ & 0.22 & NA \\
\hline D1. mean (SD) & $97(53)$ & $125(128)$ & 0.45 & NA \\
\hline D3. mean (SD) & & & & \\
\hline
\end{tabular}

SD - standard deviation; RR- Relative Risk; Cl - confidence interval; NA - not applied; BMI - Body mass index; SAPS III - Simplified Acute Physiology Score 3; SOFA - sequential organ failure assessment score; ICU - intensive care unit; $\mathrm{N}$ - number of participants; ${ }^{a} \mathrm{p}$-value from the univariate analysis. ${ }^{\mathrm{b}} \mathrm{RR}$ form the regression analysis. ${ }^{c}$ for six patients CT scan was not performed at hospital admission. 


\begin{tabular}{|c|c|c|c|c|}
\hline Variables & $\begin{array}{l}\text { Present }(n= \\
19)\end{array}$ & $\begin{array}{l}\text { Absence }(n= \\
25)\end{array}$ & $\begin{array}{l}\text { p- } \\
\text { value }^{a}\end{array}$ & RR (IC 95\%) ${ }^{b}$ \\
\hline \multicolumn{5}{|l|}{ yes, n(\%) } \\
\hline \multicolumn{5}{|l|}{ mean (SD) } \\
\hline $\begin{array}{l}\text { Mechanical Ventilation } \\
\text { yes, } n(\%)\end{array}$ & $5(26)$ & $5(20)$ & 0.67 & NA \\
\hline $\begin{array}{l}\text { Days on Mechanical Ventilation } \\
\text { mean (SD) }\end{array}$ & $9(8.1)$ & $16(6.1)$ & 0.21 & NA \\
\hline \multicolumn{5}{|l|}{ Days. mean (SD) } \\
\hline $\begin{array}{l}\text { SD - standard deviation; RR- Relat } \\
\text { index; SAPS III - Simplified Acute } \\
\text { score; ICU - intensive care unit; } N \text { - } \\
\text { form the regression analysis. }{ }^{C} \text { fol }\end{array}$ & $\begin{array}{l}\text { k; Cl - confider } \\
\text { ogy Score } 3 ; \subseteq \\
\text { ar of participal } \\
\text { tients CT scar }\end{array}$ & $\begin{array}{l}\text { interval; NA - } n \\
\text { A - sequential } \\
\text { a p-value from } \\
\text { as not perform }\end{array}$ & $\begin{array}{l}\text { pplied; } B \\
\text { n failure } \\
\text { univaria } \\
\text { t hospit }\end{array}$ & $\begin{array}{l}\text { - - Body mass } \\
\text { ssessment } \\
\text { analysis. }{ }^{\text {b }} \text { RR } \\
\text { admission. }\end{array}$ \\
\hline
\end{tabular}

The impact of these dysfunctions on the BI was measured. Both the presence of desaturation on 6MWT (mean BI $91 \pm 25$ vs $74 \pm 27$ ) and reduced VEF1 (mean BI $89 \pm 24$ vs $68 \pm 32$ ) were significantly associated with lower BI scores $(p<0.05)$. DCLO was marginally but not significantly associated with lower BI scores (mean BI $92 \pm 23$ vs $76 \pm 30, p=0.06$ ). There was no association between MIP and the extension of CT lesions and BI scores. When desaturation on 6MWT, FEV1 and DCLO entered in a linear regression only FEV1 $(p=0.019)$ and marginally DCLO $(p=0.06)$ were associated with lower Barthel scores (R square for the model 0.28).

Additionally, both the presence of desaturation on 6MWT (mean mMRC $0.8 \pm 0.8$ vs $1.7 \pm 1.4$ ) and reduced DCLO (mean mMRC $0.8 \pm 1.2$ vs $1.6 \pm 1.0$ ) were significantly associated with higher mMRC scores $(p<0.05)$. FEV1 and the extension of CT lesions were marginally, but not significantly associated with higher mMRC scores (mean mMRC $1.0 \pm 1.0$ vs $1.6 \pm 1.7, p=0.17$ for FEV1; $0.9 \pm 1.1$ vs $1.3 \pm 1.2, p=$ 0.17 for CT lesions). There was no association between MIP and mMRC scores. When desaturation on 6MWT, FEV1, DCLO, and CT lesions entered in a linear regression only FEV1 $(p=0.017)$ and marginally $\operatorname{DCLO}(p=0.12)$ were associated with higher mMRC scores (R square for the model 0.32$)$.

\section{Discussion}

Confirming our hypothesis, respiratory dysfunction six months after hospital discharge from COVID-19 was common and negatively impacted the ADL. 
Lung CT scan were abnormal in more than half of the patients, and this is consistent with other studies $(6,8)$. Interestingly, even variables such as mechanical ventilation and respiratory SOFA were not a risk factor associated with abnormal CT scan at six months. Unlike González et al. (2021) (11) demonstrated that the persistence of lung abnormalities on CT scan was associated the length of invasive mechanical ventilation. Dyspnea was attributed to abnormalities on lung CT scan (11), and this is similar to our results; half of the patients who presented lung dysfunction measured by the 6MWT, DLCP, and MIP had persistent CT scan abnormalities.

Results for abnormal lung function assessed by the 6MWT, FEV1, DLCP, and MIP showed that a considerable proportion (18 to 43\%) had at least one alteration. DLCO and MIP were the most prevalent dysfunction observed; thus, intrinsic lung function and respiratory muscle strength were affected after COVID-19. A systematic review evaluated pulmonary function after COVID-19 (12). It included three hundred eighty patients in the data synthesis. In the sensitivity analysis, the study found a prevalence of

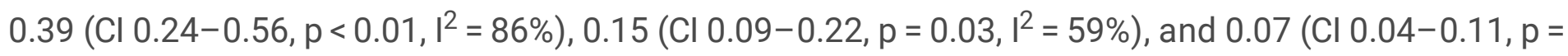
$\left.0.31, I^{2}=16 \%\right)$ for altered DLCO, restrictive pattern and obstructive pattern, respectively, consistent with our findings. As previously demonstrated $(9,10)$, an increased risk for lung dysfunction was observed in women. In our sample, female sex was an independent risk factor for decreasing 6MWT and DLCO. Additionally, this was also true to MIP in the univariate analysis. Interestingly, confirming previous reports (13), female sex was not significantly associated with persistent CT-scan abnormalities; neither is persistent CT-scan abnormalities universally present in lung dysfunction patients, suggesting that distinct mechanisms could be related to these outcomes. Further biomarkers studies could help to understand the underlying mechanisms that drive these alterations.

Other risk factors associated with lung dysfunction at 6-months that were statistically significant in the univariate analysis were Charlson comorbidity index and age. This is an exciting finding suggesting that for "Long-COVID19" premorbid characteristics are more important as a risk factor when compared to variables associated with the COVID-19 acute phase. This is different when compared to Huang et al. 2021 findings (10). They found that impaired DCLO was most prevalent in patients with more severe illnesses. The population in Huang's study included less severe COVID-19 patients when compared to our study, and this could partially explain these differences.

These dysfunctions seem to be of clinical relevance since they are associated with a decreased Barthel index and a higher degree of dyspnea assessed by the mMRC. It is important to note that the linear regression model explained $28 \%$ and $32 \%$ of the alterations in the ADL and mMRC, respectively. Thus, besides lung dysfunction, others factors associated with "Long-COVID-19" could impact ADL impairment. Huang et al. 2021(10) found that fatigue and muscle weakness were common 6-months after the onset of COVID-19 symptoms, and such symptoms could impact ADL and mMRC. Further studies should be performed to determine clinical factors associated with long-term limitations in ADL and dyspnea intensity. 
This study has several limitations. First, due to the urgent need for data, we anticipated the evaluation for these 44 patients to 6 -months after hospital discharge. The small sample size may result in falsenegative results. However, the significant associations that were found, together with the prospective design with a blind analysis, strengthen the results' credibility. Initially, these patients would be followed up only by phone interview until 12-months after hospital discharge. Follow-up keeps going, and all patients will have a complete evaluation at 12-months after hospital discharge. Second, due to epidemiologic characteristics of the pandemia, it was not possible to include SARS patients of other etiologies such as influenza.

\section{Conclusion}

Long-term lung dysfunction is relatively common in survivors from severe COVID-19 and impacts negatively on ADL and the intensity of dyspnea, similar to studies in high-income countries. These results highlight the need to develop strategies to prevent and treat the burden of disease associated with COVID19.

\section{Abbreviations}

6-minute walk test - 6MWT

Activities of daily living - ADL

Barthel index - BI

Computed tomography - CT

c-reactive protein - CRP

Forced expiratory volume in one second - FEV1

Intensive care unit - ICU

Lung carbon monoxide diffusion - DLCO

Maximum inspiratory pressure - MIP

Modified medical research council - mMRC

Sequential Organ Failure Assessment - SOFA

Simplified Acute Physiology Score - SAPS

\section{Declarations}


Ethics approval and consent to participate

The São José hospital's Institutional Review Board approved the protocol under the number 31384620.6.1001.5364. All patients or their surrogates gave written consent before inclusion in the study.

Consent for publication

Not applicable

\section{Availability of data and materials}

The datasets used and/or analysed during the current study are available from the corresponding author on reasonable request.

\section{Competing interests}

The authors declare that they have no competing interests

\section{Funding}

MCTIC/CNPq/FNDCT/MS/SCTIE/DECIT, 07/2020, grant number 401263/2020-7

BRF S.A. Hub unrestricted donation

\section{Author contributions}

G. S. P. had full access to all of the data in the study and contributed to the study design, data collection and interpretation, and writing of manuscript.. C. S. S. contributed to data collection and literature search. R. W. contributed to the study design and to writing of the manuscript. C. R. contributed to the study design and to writing of the manuscript. F.D-P, contributed to the study design, data interpretation, and writing of manuscript.

\section{References}

1. Ranzani OT, Bastos LSL, Gelli JGM, et al. Characterisation of the first 250,000 hospital admissions for COVID-19 in Brazil: a retrospective analysis of Nationwide data. Lancet Respir Med. 2021;9(4):407-18.

2. Hu B, Guo H, Zhou P, et al. Characteristics of SARS-CoV-2 and COVID-19. Nature Rev Microbiol. 2021;19(3):141-54.

3. Ramani C, Kadl A. Surviving. COVID-19: A Burden Yet to Come? Chest. 2021;160(1):15-6.

4. Wiersinga WJ, Rhodes A, Cheng AC, Peacock SJ, Prescott HC. Pathophysiology, Transmission, Diagnosis, and Treatment of Coronavirus Disease 2019 (COVID-19): A Review. JAMA. 2020;324(8):782-93. 
5. Nalbandian A, Sehgal K, Gupta A, et al. Post-acute COVID-19 syndrome. Nature Med. 2021;27(4):601-15.

6. McGroder CF, Zhang D, Choudhury MA, et al. Pulmonary fibrosis 4 months after COVID-19 is associated with severity of illness and blood leucocyte telomere length. Thorax. In press. doi: 10.1136/thoraxjnl-2021-217031.

7. McDonald LT. Cure after COVID-19: survivors are at risk for pulmonary fibrosis? Am J Physiol Lung Cell Mol Physiol. 2021;320(2):257-65.

8. Huang C, Huang L, Wang Y, et al. 6-month consequences of COVID-19 in patients discharged from hospital: a cohort study. Lancet. 2021;397(10270):220-32.

9. Wu X, Liu X, Zhou Y, et al. 3-month, 6-month, 9-month, and 12-month respiratory outcomes in patients following COVID-19-related hospitalisation: a prospective study. Lancet Respir Med. 2021;9(7):74754.

10. Huang L, Yao Q, Gu X, et al. 1-year outcomes in hospital survivors with COVID-19: a longitudinal cohort study. Lancet. 2021;398(10302):747-58.

11. González J, Benítez ID, Carmona P, et al. CIBERESUCICOVID Project (COV20/00110, ISCIII). Pulmonary function and radiologic features in survivors of critical COVID-9: a 3-month prospective cohort. Chest. 2021;160(1):187-98.

12. Sanchez-Ramirez DC, Normand K, Zhaoyun Y, Torres-Castro R. Long-Term Impact of COVID-19: A Systematic Review of the Literature and Meta-Analysis. Biomedicines. 2021;9(8):900.

13. TheWriting Committee for the COMEBAC Study Group. Four-Month Clinical Status of a Cohort of Patients After Hospitalization for COVID-19. JAMA. 2021;325(15):1525-34. 